\section{P47 A SYSTEMATIC SCOPING REVIEW OF COMMUNITY- BASED INTERVENTIONS FOR THE PREVENTION OF POOR MENTAL HEALTH IN OLDER ADULTS}

\begin{abstract}
${ }^{1,2}{ }^{2}$ Lee $^{*},{ }^{3} \mathrm{M}$ McGrath, ${ }^{1} \mathrm{O}$ Remes, ${ }^{3,4}$ J Dyxhoorn, ${ }^{5} \mathrm{~S}$ Gnani, ${ }^{4} \mathrm{EJ}$ Oliver, ${ }^{3,6} \mathrm{D}$ Osborne, ${ }^{4} \mathrm{~K}$ Walters, ${ }^{1} \mathrm{~L}$ Lafortune, ${ }^{7} \mathrm{I}$ Kuhn, ${ }^{8} \mathrm{~F}$ Duncan, ${ }^{5} \mathrm{C}$ Baskin, ${ }^{9} \mathrm{E}$ Kaner, ${ }^{3} \mathrm{JB}$ Kirkbride. ${ }^{1}$ Cambridge Institute of Public Health, CIPH, Cambridge, UK; ${ }^{2}$ Cambridge Institute for Sustainability Leadership, Cambridge, UK; ${ }^{3}$ Division of Psychiatry, University College London, London, UK; ${ }^{4}$ Department of Primary Care and Population Health, University College London, Royal Free Campus, London, UK; ${ }^{5}$ Department of Primary Care and Public Health, School of Public Health, Imperial College London, London, UK; ${ }^{6}$ Camden and Islington NHS Foundation Trust, London, UK; ${ }^{7}$ University of Cambridge Medical Library, School of Clinical Medicine, Cambridge Biomedical Campus, Cambridge, UK; ${ }^{8}$ Department of Sport and Exercise Sciences, Durham University, Durham, UK; ${ }^{9}$ Population Health Sciences Institute, Newcastle upon Tyne, UK
\end{abstract}

\subsection{6/jech-2020-SSMabstracts. 141}

Background Mental health in older adults is often overlooked, or pathologised into dementias and brain diseases. Yet, the spectrum of mental health is as varied as at other life stages, with additional risks to mental health presented by physical decline, onset of ill health, reducing income, social isolation and bereavement. Community interventions can reduce the impact of these, potentially protecting mental health and promoting wellbeing. There is a need to understand: what kind of interventions, for which stressors, prevail in the UK setting; which demonstrate effectiveness, for whom, and how; and, where there may be intervention gaps or need for focused research.

We conducted a scoping review of community-based interventions in the UK, to improve the mental health and wellbeing of older adults, with a focus on psychosocial adversity.

Methods We searched electronic databases to identify academic studies of community interventions which aim to improve mental health and wellbeing outcomes for older adults, extracted data, and conducted a narrative synthesis. Community interventions were broadly defined as any non-clinical intervention that aimed to improve psychosocial aspects of participants lives, and extended to social prescribing and assetoriented initiatives. This could cover a range of programmes and initiatives from providing mental health-related information, support and advice, broader community engagement, building social connections, mobilising resources.

Results From the 902 studies returned from database searches and 5 studies identified through manual bibliography searching, 889 full-text articles were assessed for eligibility and 56 included in the final data synthesis. Thirteen initial categories of community-based intervention were identified, including: befriending; peer support; group support; creative/cultural activities; welfare advice; social prescribing and asset-based community interventions.

Conclusion The evidence base for effective and cost-effective community public mental health interventions for older adults is mixed and relatively weak. Evidence can be found. e.g. in favour of befriending and in cultural activities, though not always backed by a good understanding of who precisely benefits, and for how long. As a whole, and even within category, strong conclusions are hampered by inconsistent outcome measures, small samples, and lack of follow up. Useful insights in implementation are however presented by interventions with an 'upstream' and very localised approach, reflecting more recent directions in policy and practice.

We argue both for greater consistency in concept and outcome measurement, and for inclusion of theory and systemsbased approaches to evaluating effectiveness to reflect the complexity of community-based interventions and strategies and goals of reducing inequalities.

This study/project is funded by the National Institute for Health Research (NIHR) School for Public Health Research (Grant Reference Number PD-SPH-2015). The views expressed are those of the author(s) and not necessarily those of the NIHR or the Department of Health and Social Care.

\section{P48 BARRIERS AND ENABLERS TO SCREENING AND DIAGNOSING DEPRESSION AND DIABETES DISTRESS IN PEOPLE WITH TYPE 2 DIABETES MELLITUS; A QUALITATIVE EVIDENCE SYNTHESIS}

${ }^{1} \mathrm{NM}$ McGrath*, ${ }^{1} \mathrm{PM}$ Kearney, ${ }^{1} \mathrm{~S}$ McHugh, ${ }^{2} \mathrm{E}$ Toomey. ${ }^{1}$ School of Public Health, University College Cork, Cork, Ireland; ${ }^{2}$ School of Psychology, National University of Ireland Galway, Galway, Ireland

\subsection{6/jech-2020-SSMabstracts. 142}

Background Screening for depression and diabetes distress in people with type 2 diabetes (T2DM) in primary care is increasingly recommended but implementation in practice is suboptimal. As health care professionals influence detection practices, their perceptions and experiences of screening recommendations can improve understanding of aspects that work, and those which are difficult to implement. We aim to synthesise the available qualitative evidence on healthcare professionals' perceived barriers and enablers to recognising possible depression and diabetes distress in people with T2DM using validated screening tools compared to clinical questioning or no screening.

Methods Primary qualitative and mixed method studies were identified using systematic database searching of six databases and supplementary searching. We selected 'best-fit framework synthesis' to synthesise primary data using the RETREAT (Review question-Epistemology-Time/Timescale-ResourcesExpertise-Audience and purpose-Type of Data) framework. We selected the theoretical domains framework (TDF) as the a priori best fit framework as the TDF is derived from existing behaviour change theories. Quality appraisal of primary studies and confidence in the overall review findings will be determined using the CASP (Critical-Appraisal-Skills-Programme) and the GRADE-CERQual (Grading-of-RecommendationsAssessment-Development-and-Evaluation-Confidence-in-the-Evidence-from-Reviews-of-Qualitative-research) respectively. The study is registered on the international Prospective Register of Systematic Reviews (PROSPERO; registration number: CRD42019145483).

Results Ten primary qualitative studies were identified from five countries; England $(n=4)$, China $(n=1)$, the Netherlands $(n=3)$, Australia $(n=1)$, Canada $(n=1)$. No studies on detection of diabetes-distress were identified. Data extraction and analysis is ongoing. Tentative findings indicate that primary care health professionals experience barriers and enablers to detection unique in T2DM populations; symptom overlap, perceptions of role and responsibilities, the perceived value of screening in the T2DM population, and integrating screening protocols into T2DM review visits. Additional barriers to depression screening in the T2DM population; mental health stigma, patient-clinician relationship, were pertinent to depression screening in primary care populations more generally. Discussion Findings may (1) improve understanding of how depression can be more appropriately identified in people with T2DM in primary care settings from a health care 